\title{
Effects of increased body weight and short-term weight loss on serum PCSK9 levels - a prospective pilot study
}

Theodosios D. Filippatos ${ }^{1}$, Evangelos Liberopoulos ${ }^{1}$, Maria Georgoula ${ }^{1}$, Constantinos C. Tellis ${ }^{2}$, Alexandros D. Tselepis², Moses Elisaf ${ }^{1}$

\author{
${ }^{1}$ Department of Internal Medicine, School of Medicine, University of Ioannina, \\ Ioannina, Greece \\ ${ }^{2}$ Laboratory of Biochemistry, Department of Chemistry, University of Ioannina, \\ Ioannina, Greece
}

Submitted: 26 June 2017

Accepted: 31 July 2017

Arch Med Sci Atheroscler Dis 2017; 2: e46-e51

DOI: https://doi.org/10.5114/amsad.2017.70502

Copyright (c) 2017 Termedia \& Banach

\section{Abstract}

Introduction: Proprotein convertase subtilisin/kexin type 9 (PCSK9) is associated with low-density lipoprotein (LDL) catabolism, but its serum concentration is not uniformly associated with cardiovascular disease in clinical studies. Obesity is linked with increased cardiovascular risk, but the effect of increased body weight and short-term weight loss on serum PCSK9 levels is not well studied.

Material and methods: The aim of this prospective pilot study was to assess differences in serum PCSK9 levels (determined with a quantitative sandwich enzyme immunoassay) between otherwise healthy drug-naïve obese subjects and healthy individuals with normal body weight. Additionally, PCSK9 levels were determined at baseline and after a 3-month weight-loss program with a low-fat diet in a randomly assigned subgroup of the obese subjects $(n=15)$.

Results: Obese subjects ( $n=35$ ) were older (age: $43 \pm 11$ years) and had significantly higher body mass index, total cholesterol, triglycerides, LDL cholesterol (LDL-C), apolipoprotein B and homeostasis model assessment of insulin resistance (HOMA) index levels, as well as significantly lower high-density lipoprotein cholesterol (HDL-C) concentration, compared with normal-weight subjects ( $n=20$, age: $35 \pm 6$ years). Serum PCSK9 levels were significantly higher in obese subjects compared with normal-weight individuals, even after adjustment for age, LDL-C, triglycerides, HDL-C, apolipoprotein $A 1$, apolipoprotein $B$, apolipoprotein E, glucose, insulin and HOMA index levels $(p=0.018)$. Obese subjects experienced significant weight loss (from $109 \pm 22$ to $104 \pm 23 \mathrm{~kg}, p<0.01$ ), but serum PCSK9 levels did not significantly change after the 3-month weight-loss program.

Conclusions: Serum PCSK9 levels are higher in obese subjects than in normal-weight individuals. Short-term weight loss with a low-fat diet does not significantly affect PCSK9 levels.

Key words: proprotein convertase subtilisin/kexin type 9, obesity, weight loss, triglycerides, apolipoprotein.

\section{Introduction}

Obesity has reached epidemic proportions linked with deleterious health and socio-economic consequences [1]. More than one-third of the US adults is obese (body mass index (BMI) $\geq 30 \mathrm{~kg} / \mathrm{m}^{2}$ ) having signifi-

\author{
Corresponding author: \\ Prof. Moses Elisaf MD, PhD \\ Department of \\ Internal Medicine \\ School of Medicine \\ University of loannina \\ Panepistimiou Avenue \\ 45110 loannina, Greece \\ Phone: +30 2651007509 \\ E-mail: melisaf54@gmail.com
}


cantly higher annual medical costs compared with normal-weight individuals [2]. The ATTICA study cohort has also showed increasing prevalence rates of overweight and obesity in Greece [3, 4]. In parallel to these obesity trends, cardiovascular disease (CVD) incidence remains high, constituting a major public health problem with a serious economic impact [5]. Dyslipidemia constitutes one of the key CVD risk factors; thus, it is important to assess factors affecting lipid levels in obese patients as possible therapeutic targets.

Proprotein convertase subtilisin/kexin type 9 (PCSK9) is a key protein in lipid and lipoprotein metabolism that plays a principal role in the hepatic low-density lipoprotein (LDL) receptors' degradation and consequently affects LDL particles' catabolism and serum LDL cholesterol (LDL-C) levels [6]. PCSK9 also appears to interact with receptors other than the LDL receptor [7]. Gain-of-function mutations of the PCSK9 gene are identified as a cause of familial hypercholesterolemia, whereas loss-of-function mutations are associated with significantly reduced levels of LDL-C and a lower lifetime CVD risk [7, 8]. However, clinical studies regarding the role of circulating PCSK9 level on predicting CVD risk are not always consistent. A prospective cohort study showed that serum PCSK9 concentration is associated with future CVD risk even after adjustments for established CVD risk factors [9]. In contrast, a study in patients with acute coronary syndrome showed that high baseline PCSK9 plasma levels did not predict mortality at 1 year $[9,10]$. Generally, more research is needed to determine the factors that affect circulating PCSK9 concentration and its association with CVD risk. In this context, the available data regarding the effects of adiposity and weight loss on circulating PCSK9 levels are limited.

We herein present the preliminary results of a prospective pilot study that assessed: i) possible differences in serum PCSK9 levels between otherwise healthy drug-naïve obese subjects and healthy individuals with normal body weight, and ii) the effects of a 3-month weight-loss program on serum PCSK9 levels.

\section{Material and methods}

\section{Participants}

Individuals attending the Outpatient Obesity and Lipid Clinic of the University Hospital of loannina, Greece were recruited for the study. The study group included consecutive subjects with $\mathrm{BMI}>30 \mathrm{~kg} / \mathrm{m}^{2}$, whereas the control group included consecutive subjects with $\mathrm{BMI}<25 \mathrm{~kg} / \mathrm{m}^{2}$ (in Greece, people have the option to attend hospital clinics without external referral in order to assess their lipid profile and other cardiovascular risk fac- tors). Exclusion criteria were: (i) abnormal hepatic function (aminotransferase activity $>3$ times the upper limit of normal, and/or history of chronic liver disease, such as cirrhosis or alcoholic liver disease), (ii) chronic kidney disease (estimated glomerular filtration rate $<60 \mathrm{ml} / \mathrm{min} / 1.73 \mathrm{~m}^{2}$ ), (iii) diabetes (fasting blood glucose $>7.0 \mathrm{mmol} / \mathrm{l}$; $126 \mathrm{mg} / \mathrm{dl}$, (iv) raised thyroid-stimulating hormone (TSH) levels (> $5.0 \mu \mathrm{U} / \mathrm{ml}$ ) or other endocrinopathy, and (v) any medication.

All participants gave their informed consent and the study protocol was approved by the institutional ethics committee.

\section{Dietary habits and intervention}

Adherence to the Mediterranean diet was evaluated with the MedDietScore (range: 0-55; higher values indicate greater adherence) [11].

A randomly assigned subgroup of patients with obesity $(n=15)$ received dietary intervention. At baseline a dietician, based on each patient's basal energy requirements and estimation of the subject's typical activity level, prescribed an individualized low-fat diet, promoting an approximately $500 \mathrm{kcal}$ reduction in daily energy intake. All participants completed 4-day diet records at baseline and at the end of treatment. The administered diets consisted of a mean of $1512 \pm 406$ $\mathrm{kcal} /$ day (ranging from 1085 to $2000 \mathrm{kcal} /$ day depending on the initial body weight). The daily distribution of nutrients during the study was: carbohydrates $52.9 \pm 4.0 \%$, fat $28.1 \pm 3.3 \%$ (monounsaturated $15.1 \pm 1.6 \%$, polyunsaturated $7.4 \pm 1.6 \%$ and saturated fatty acids $5.4 \pm 0.9 \%$ ) and protein $19.0 \pm 1.6 \%$. All patients were asked to attend the clinic monthly during the treatment in order to assess diet compliance. Food diaries and discussion during visits were used to ensure dietary compliance throughout the study period. Every month the dietician provided instructions based on dietary intake-recording procedures as part of a behavior-modification program.

\section{Anthropometric variables}

Height, without shoes, was measured to the nearest $0.5 \mathrm{~cm}$ and body weight to the nearest $100 \mathrm{~g}$, without shoes and with light clothing. BMI was calculated as weight (in kilograms) divided by the height (in meters) squared. Waist circumference was measured at the end of a normal expiration by placing a tape in a horizontal plane around the abdomen at the level of the iliac crest.

\section{Biochemical parameters}

All laboratory determinations were carried out after an overnight fast. Serum concentrations of fasting glucose, total cholesterol, high-density li- 
poprotein cholesterol (HDL-C) and triglycerides were determined enzymatically on an Olympus AU600 clinical chemistry analyzer (Olympus Diagnostica, Hamburg, Germany). LDL-C was calculated using the Friedewald formula (all participants had triglycerides $<400 \mathrm{mg} / \mathrm{dl}$ ). Serum apolipoprotein levels were measured with a Behring Holding $\mathrm{GmbH}$ analyzer (Liederbach, Germany). Fasting serum insulin levels were measured by an AxSYM insulin assay microparticle enzyme immunoassay on an AzSYM analyzer (Abbott Diagnostics, Illinois, USA). Finally, the homeostasis of model assessment (HOMA) index was calculated as follows: fasting insulin $(\mathrm{mU} / \mathrm{l})$ * fasting glucose $(\mathrm{mg} /$ dl)/405.

\section{Serum PCSK9 measurement}

Serum PCSK9 concentration was determined by a quantitative sandwich enzyme immunoassay technique [12]. Briefly, standards and samples are pipetted into wells pre-coated with a monoclonal antibody specific for human PCSK9 and any PCSK9 present is bound by the immobilized antibody. After washing away any unbound substances, an enzyme-linked polyclonal antibody specific for human PCSK9 is added to the wells. Following a wash to remove any unbound antibody-enzyme reagent, a substrate solution is added to the wells and color develops in proportion to the amount of PCSK9 bound in the initial step. The intensity of the color is measured using a microplate reader set to $450 \mathrm{~nm}$.

The intra-assay coefficient of variation ranges from 4.1 to 6.5 and the inter-assay coefficient of variation ranges from 4.1 to 6.0 [12].

\section{Statistical analysis}

Our primary hypothesis was that patients with obesity would have significantly different serum PCSK9 levels compared with individuals with normal weight. The secondary hypothesis was that short-term weight loss would affect serum PCSK9 concentration. This is a pilot study and therefore no formal power calculations were performed. Values are given as mean \pm standard deviation (SD). Variables were tested for normality with the Kolmogorov-Smirnov test. The $\chi^{2}$ test was used to compare categorical variables. Possible differences between the two study groups were assessed with the independent-samples $t$-test or Mann-Whitney test for parametric and non-parametric variables, respectively. Univariate analysis of the general linear model was used to compare PCSK9 levels between the two groups using as covariates age, lipid/lipoprotein and carbohydrate metabolism variables. The paired-samples $t$-test or Wilcoxon signed-rank test for parametric and non-parametric variables, respectively, was used to assess the effects of the weight-loss program. The correlations between variables were assessed using Pearson's correlation coefficient $(r)$ or Spearman's correlation coefficient $\left(r_{s}\right)$ for parametric and non-parametric variables, respectively. Significance was defined as $p<0.05$ (two-tailed). Analyses were performed using the SPSS 21.0 statistical package (SPSS Inc.).

\section{Results}

A total of 35 patients with obesity and 20 controls were enrolled. Patients with obesity had significantly higher age, body weight, BMI, waist circumference, total cholesterol, triglycerides, LDL-C, apolipoprotein B, glucose, insulin, and HOMA index, whereas they had significantly lower HDL-C, apolipoprotein A1 and MedDietScore, compared with the control group (Table I). Serum PCSK9 levels were significantly higher in patients with obesity compared with the control group $(p<0.01)$. This difference remained significant even after adjustment for age, LDL-C, triglycerides, HDL-C, apolipoprotein $\mathrm{A} 1$, apolipoprotein $\mathrm{B}$, apolipoprotein $E$, glucose, insulin and HOMA index levels $(p=0.018)$. Notably, when BMI was used as a covariate, the difference between the two groups was non-significant ( $p=0.53$ ).

Since the two groups had significantly different age, we performed partial correlations in the total sample adjusting for age. We observed a significant age-adjusted correlation between PCSK9 levels and BMI $\left(r_{s}=0.36, p=0.02\right)$, body weight $\left(r_{s}=\right.$ $0.35, p=0.03)$ and waist circumference $\left(r_{s}=0.36\right.$, $p=0.04)$. We also observed a negative age-adjusted correlation between PCSK9 levels and MedDietScore $\left(r_{s}=-0.32, p=0.045\right)$. No significant association between PCSK9 concentration and lipid/ lipoprotein or carbohydrate metabolism variables was observed.

Significant weight loss was observed in the subgroup of patients with obesity who were enrolled in the 3-month dietary intervention (Table II). This effect was accompanied by a significant improvement in triglycerides and apoE levels, as well as numeric improvements of marginal significance in HOMA index. Serum PCSK9 levels did not significantly change compared with baseline after the 3-month dietary intervention (Table II and Figure 1). We did not observe any significant association between the percent changes in PCSK9 levels and the changes in other lipid or carbohydrate metabolism variables (data not shown).

\section{Discussion}

This prospective pilot study assessed serum PCSK9 levels in patients with obesity at baseline and after short-term weight loss. To the best of our 
Table I. Anthropometric and metabolic variables in obese subjects and the control group

\begin{tabular}{|c|c|c|c|}
\hline Parameter & Obese $(n=35)$ & Controls $(n=20)$ & $P$-value \\
\hline Age [years] & $43 \pm 11$ & $35 \pm 6$ & 0.01 \\
\hline Gender (males/females) & $11 / 24$ & $6 / 14$ & NS \\
\hline Body weight [kg] & $114.7 \pm 31.9$ & $64.1 \pm 10.5$ & $<0.001$ \\
\hline $\mathrm{BMI}\left[\mathrm{kg} / \mathrm{m}^{2}\right]$ & $39.7 \pm 7.8$ & $22.7 \pm 2.0$ & $<0.001$ \\
\hline Waist circumference $[\mathrm{cm}]$ & $124 \pm 19$ & $84 \pm 9$ & $<0.001$ \\
\hline Total cholesterol [mg/dl] & $212 \pm 29$ & $172 \pm 27$ & $<0.001$ \\
\hline Triglycerides [mg/dl] & $125 \pm 60$ & $67 \pm 32$ & $<0.001$ \\
\hline $\mathrm{HDL}-\mathrm{C}[\mathrm{mg} / \mathrm{dl}]$ & $53 \pm 12$ & $57 \pm 11$ & 0.04 \\
\hline LDL-C [mg/dl] & $134 \pm 24$ & $102 \pm 21$ & $<0.001$ \\
\hline apoB [mg/dl] & $89 \pm 17$ & $65 \pm 15$ & $<0.001$ \\
\hline apoA1 [mg/dl] & $143 \pm 23$ & $159 \pm 23$ & 0.048 \\
\hline apoE $[\mathrm{mg} / \mathrm{l}]$ & $43 \pm 9$ & $37 \pm 15$ & NS \\
\hline Glucose $[\mathrm{mg} / \mathrm{dl}]$ & $92 \pm 10$ & $80 \pm 8$ & $<0.001$ \\
\hline Insulin $[\mu \mathrm{U} / \mathrm{ml}]$ & $13.2 \pm 7.5$ & $5.5 \pm 2.1$ & $<0.001$ \\
\hline HOMA index & $3.0 \pm 1.6$ & $1.1 \pm 0.5$ & $<0.001$ \\
\hline MedDietScore & $17 \pm 3$ & $25 \pm 4$ & $<0.001$ \\
\hline PCSK9 [ng/ml] & $229 \pm 65$ & $156 \pm 43$ & $<0.001^{*}$ \\
\hline
\end{tabular}

Values are given as mean $\pm S D . B M I-$ body mass index, HDL-C - high-density lipoprotein cholesterol, $L D L-C$ - low-density lipoprotein cholesterol, apo - apolipoprotein, HOMA - homeostasis model assessment, PCSK9 - proprotein convertase subtilisin/kexin type 9.

Table II. Anthropometric and metabolic variables at baseline and after 3 months in obese subjects $(n=15 ; 5$ males, 10 females)

\begin{tabular}{|c|c|c|c|}
\hline Parameter & Baseline & 3 months & $P$-value \\
\hline Body weight [kg] & $109 \pm 22$ & $104 \pm 23$ & $<0.001$ \\
\hline BMI $\left[\mathrm{kg} / \mathrm{m}^{2}\right]$ & $38 \pm 5$ & $36 \pm 5$ & $<0.001$ \\
\hline Total cholesterol [mg/dl] & $211 \pm 32$ & $204 \pm 32$ & NS \\
\hline Triglycerides [mg/dl] & $126 \pm 59$ & $91 \pm 33$ & $<0.01$ \\
\hline HDL cholesterol [mg/dl] & $51 \pm 12$ & $50 \pm 11$ & NS \\
\hline LDL cholesterol [mg/dl] & $135 \pm 26$ & $136 \pm 26$ & NS \\
\hline Apolipoprotein B [mg/dl] & $92 \pm 17$ & $84 \pm 21$ & NS \\
\hline Apolipoprotein A1 [mg/dl] & $139 \pm 21$ & $129 \pm 20$ & NS \\
\hline Apolipoprotein E [mg/l] & $43 \pm 6$ & $38 \pm 9$ & 0.04 \\
\hline Glucose [mg/dl] & $96 \pm 10$ & $93 \pm 8$ & NS \\
\hline Insulin [mU/ml] & $12.9 \pm 3.6$ & $10.0 \pm 3.2$ & NS \\
\hline HOMA index & $3.2 \pm 0.9$ & $2.3 \pm 0.7$ & 0.07 \\
\hline PCSK9 [ng/ml] & $207 \pm 54$ & $218 \pm 57$ & NS \\
\hline
\end{tabular}

Values are given as mean $\pm S D$. BMI - body mass index, HDL-C - high-density lipoprotein cholesterol, LDL-C - low-density lipoprotein cholesterol, HOMA - homeostasis model assessment, PCSK9-proprotein convertase subtilisin/kexin type 9.

knowledge this is the first study to examine the effects of short-term weight loss on serum PCSK9 concentration. The main findings were that drugnaive patients with obesity had significantly higher serum PCSK9 levels compared with subjects with normal weight, but short-term weight loss did not significantly change PCSK9 concentration.

PCSK9 is associated with increased hepatic LDL receptor degradation and elevated serum LDL-C levels [6]. Previous studies have shown a large variability of circulating serum PCSK9 concentrations, which ranged up to 100 -fold in the general 


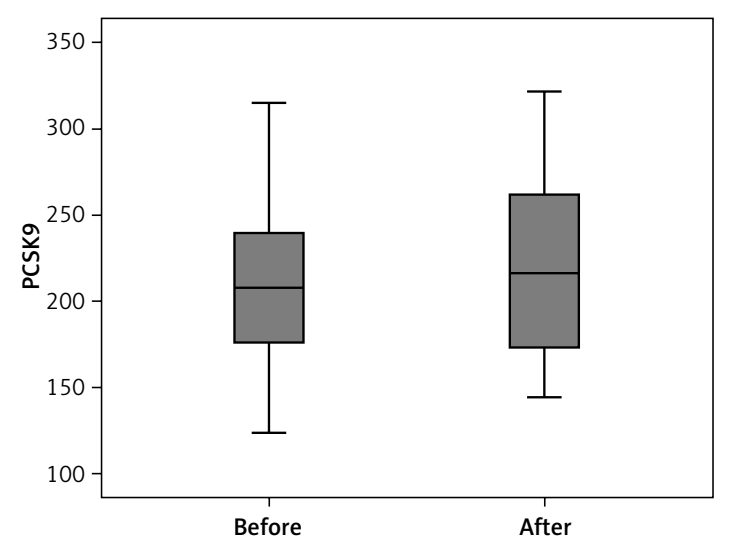

Figure 1. Alterations of serum proprotein convertase subtilisin/kexin type 9 (PCSK9) levels during the 3-month weight-loss program. Values are given in $\mathrm{ng} / \mathrm{ml}$

population [13]. The variability of serum PCSK9 levels indicates that many factors may affect them, possibly leading to different associations with cardiovascular risk $[9,10]$. We observed a significantly higher PCSK9 concentration in patients with obesity, which remained significant even after adjustment for age, lipid/lipoprotein and carbohydrate metabolism variables. Addition of BMI in the multivariate analysis resulted in a non-significant serum PCSK9 difference between subjects with obesity and the control group, which indicates that adiposity is a contributing factor affecting PCSK9 levels in our population. This inference is also supported by the significant age-adjusted correlation of baseline PCSK9 concentration with adiposity indexes. Increased adiposity is associated with changes in many metabolic pathways, and is linked with alterations in adipokine production and circulation [1420]. Future studies should aim to assess possible mechanisms for the obesity-associated increase in PCSK9 levels, for example a possible mediating or contributing effect of liver fat accumulation [21].

Previous reports have shown a relatively weak correlation of LDL-C levels with serum PCSK9 levels $(r=0.24)$ in the general population that explained less than $8 \%$ of the variation in LDL-C levels [13]. In obese subjects, serum PCSK9 concentrations correlated significantly with serum LDL-C concentration, but not with VLDL-triglyceride secretion or clearance rate [18]. Additionally, relatively weak correlations of PCSK9 levels with triglycerides, insulin and glucose have been reported [13, 22]. We did not find a significant age-adjusted correlation between PCSK9 and LDL-C concentration or other lipid/carbohydrate variables, an observation that may be due to the small sample size. It should be mentioned that the intact fraction of PCSK9 is active, but the furin-cleaved fraction regulates LDL receptor and serum cholesterol levels less efficiently than intact PCSK9 [23]. In the present study total serum PCSK9 levels (containing both PCSK9 fractions) were measured; thus this also may explain the poor correlation of serum PCSK9 with LDL-C concentration. A recent study also did not report a significant association between PCSK9 and LDL-C levels [24], whereas another study showed a positive correlation between PCSK9 and LDL-C concentration only in men [25].

We did not observe any significant change in serum PCSK9 levels after the 3-month weightloss program. A previous study showed that a 20 week weight-loss program in the context of the Mediterranean diet did not significantly change serum PCSK9 levels in men with metabolic syndrome [26]. Thus, it seems that weight loss per se does not alter circulating PCSK9 levels. In contrast, dietary habits seem to affect serum PCSK9 concentration. For example, in mice, PCSK9 mRNA quantity is decreased by $73 \%$ after $24 \mathrm{~h}$ of fasting and restored upon high carbohydrate refeeding [27]. PCSK9 concentrations increase on a shortterm high-fructose diet [28]. Additionally, previous studies have shown that a Mediterranean diet reduces circulating PCSK9 levels [26]. A Mediterranean diet is a dietary pattern associated with a reduction of diabetes and CVD incidence [29]. Our results showing a negative association between PCSK9 levels and adherence to the Mediterranean diet (assessed by the MedDietScore) also support the notion that the Mediterranean diet is associated with decreased PCSK9 levels. Our study used a low-fat diet and the effect of the changes in diet composition on serum PCSK9 levels could not be assessed. Thus, future studies need to assess different dietary compositions with or without weight loss in order to elucidate their effects on circulating PCSK9 concentration.

This pilot study has certain limitations. We included a rather small sample that possibly obscured correlations between variables and did not permit subgroup investigations. Furthermore, this study did not assess the effect of weight loss with different dietary compositions on PCSK9 levels. It is possible that a greater number of patients, a longer duration of hypocaloric diet and clinically significant body weight loss are needed to establish the change in serum PCSK9 levels. Additionally, local PCSK9 levels and not those circulating in the blood might play a significant role in LDL particle metabolism.

In conclusion, this pilot study shows increased serum PCSK9 levels in subjects with obesity that do not significantly change with short-term weight loss with a low-fat diet.

\section{Conflict of interest}

This study was conducted independently. MSE reports personal fees from Astra-Zeneca, grants 
and personal fees from MSD, personal fees from PFIZER, ABBOTT, Sanofi-Aventis, Boehringer Ingelheim, Eli-Lilly and GSK. ELN has participated in advisory committees, clinical studies and lectures sponsored by Astra-Zeneca, Boehringer Ingelheim, MSD, Novartis, Novo Nordisk, Sanofi-Aventis, Eli-Lilly, Mylan and AMGEN. TDF and ADT have given talks and attended conferences sponsored by various pharmaceutical companies, including Bristol-Myers Squibb, Pfizer, Lilly, Abbott, Amgen, AstraZeneca, Novartis, Vianex, Teva and MSD.

\section{References}

1. Ng M, Fleming T, Robinson M, et al. Global, regional, and national prevalence of overweight and obesity in children and adults during 1980-2013: a systematic analysis for the Global Burden of Disease Study 2013. Lancet 2014; 384: 766-81.

2. Finkelstein EA, Trogdon JG, Cohen JW, Dietz W. Annual medical spending attributable to obesity: payer-and service-specific estimates. Health Aff (Millwood) 2009; 28: w822-31.

3. Panagiotakos DB, Pitsavos C, Chrysohoou C, et al. Epidemiology of overweight and obesity in a Greek adult population: the ATTICA Study. Obes Res 2004; 12: 1914-20.

4. Yannakoulia M, Panagiotakos D, Pitsavos C, et al. Fiveyear incidence of obesity and its determinants: the ATTICA study. Public Health Nutr 2009; 12: 36-43.

5. Mozaffarian D, Benjamin EJ, Go AS, et al. Heart Disease and Stroke Statistics-2016 Update: A Report From the American Heart Association. Circulation 2016; 133: e38-360.

6. Horton JD, Cohen JC, Hobbs HH. Molecular biology of PCSK9: its role in LDL metabolism. Trends Biochem Sci 2007; 32: 71-7.

7. Dixon DL, Trankle C, Buckley L, et al. A review of PCSK9 inhibition and its effects beyond LDL receptors. J Clin Lipidol 2016; 10: 1073-80.

8. Cohen JC, Boerwinkle E, Mosley TH Jr, Hobbs HH. Sequence variations in PCSK9, low LDL, and protection against coronary heart disease. N Engl J Med 2006; 354: 1264-72.

9. Gencer B, Montecucco F, Nanchen D, et al. Prognostic value of PCSK9 levels in patients with acute coronary syndromes. Eur Heart J 2016; 37: 546-53.

10. Leander K, Malarstig A, Van't Hooft FM, et al. Circulating proprotein convertase subtilisin/kexin type 9 (PCSK9) predicts future risk of cardiovascular events independently of established risk factors. Circulation 2016; 133: 1230-9.

11. Panagiotakos DB, Pitsavos C, Stefanadis C. Dietary patterns: a Mediterranean diet score and its relation to clinical and biological markers of cardiovascular disease risk. Nutr Metab Cardiovasc Dis 2006; 16: 559-68.

12. Human Proprotein Convertase 9/PCSK9 Quantikine ELISA Kit. R\&D Systems. https://resources.rndsystems. com/pdfs/datasheets/dpc900.pdf. Accessed at 07-062017.

13. Lakoski SG, Lagace TA, Cohen JC, Horton JD, Hobbs HH. Genetic and metabolic determinants of plasma PCSK9 levels. J Clin Endocrinol Metab 2009; 94: 2537-43.

14. Klop B, Elte JW, Cabezas MC. Dyslipidemia in obesity: mechanisms and potential targets. Nutrients 2013; 5: 1218-40.
15. Seifalian AM, Filippatos TD, Joshi J, Mikhailidis DP. Obesity and arterial compliance alterations. Curr Vasc Pharmacol 2010; 8: 155-68.

16. Esser N, Legrand-Poels S, Piette J, Scheen AJ, Paquot N. Inflammation as a link between obesity, metabolic syndrome and type 2 diabetes. Diabetes Res Clin Pract 2014; 105: 141-50.

17. Filippatos TD, Randeva HS, Derdemezis CS, Elisaf MS, Mikhailidis DP. Visfatin/PBEF and atherosclerosis-related diseases. Curr Vasc Pharmacol 2010; 8: 12-28.

18. Sullivan S, Fabbrini E, Horton JD, Korenblat K, Patterson BW, Klein S. Lack of a relationship between plasma PCSK9 concentrations and hepatic lipoprotein kinetics in obese people. Transl Res 2011; 158: 302-6.

19. Li S, Xu RX, Zhang Y, et al. Relation of resistin to proprotein convertase subtilisin-kexin type 9 levels in coronary artery disease patients with different nutritional status. J Endocrinol Invest 2015; 38: 1291-9.

20. Kwakernaak AJ, Lambert G, Muller Kobold AC, Dullaart RP. Adiposity blunts the positive relationship of thyrotropin with proprotein convertase subtilisin-kexin type 9 levels in euthyroid subjects. Thyroid 2013; 23: 166-72.

21. Ruscica M, Ferri N, Macchi C, et al. Liver fat accumulation is associated with circulating PCSK9. Ann Med 2016; 48: 384-91.

22. Ibarretxe D, Girona J, Plana N, et al. Circulating PCSK9 in patients with type 2 diabetes and related metabolic disorders. Clin Investig Arterioscler 2016; 28: 71-8.

23. Lipari MT, Li W, Moran P, et al. Furin-cleaved proprotein convertase subtilisin/kexin type 9 (PCSK9) is active and modulates low density lipoprotein receptor and serum cholesterol levels. J Biol Chem 2012; 287: 43482-91.

24. Tecson KM, Panettiere-Kennedy KS, Won JI, Garg P, Olugbode $\mathrm{O}$, McCullough PA. Relation between proprotein convertase subtilisin/kexin type 9 and directly measured low-density lipoprotein cholesterol. Proc (Bayl Univ Med Cent) 2017; 30: 16-20.

25. Mayne J, Raymond A, Chaplin A, et al. Plasma PCSK9 levels correlate with cholesterol in men but not in women. Biochem Biophys Res Commun 2007; 361: 451-6.

26. Richard C, Couture P, Desroches S, et al. Effect of the Mediterranean diet with and without weight loss on surrogate markers of cholesterol homeostasis in men with the metabolic syndrome. Br J Nutr 2012; 107: 705-11.

27. Costet P, Cariou B, Lambert G, et al. Hepatic PCSK9 expression is regulated by nutritional status via insulin and sterol regulatory element-binding protein 1c. J Biol Chem 2006; 281: 6211-8.

28. Cariou B, Langhi C, Le Bras M, et al. Plasma PCSK9 concentrations during an oral fat load and after short term high-fat, high-fat high-protein and high-fructose diets. Nutr Metab (Lond) 2013; 10: 4.

29. Filippatos TD, Panagiotakos DB, Georgousopoulou EN, et al. Mediterranean diet and 10-year (2002-2012) incidence of diabetes and cardiovascular disease in participants with prediabetes: the ATTICA study. Rev Diabet Stud 2016; 13: 226-35. 